\title{
Strongly Coupled Fermions in Nature and the Laboratory
}

\section{J. Carlson*, Alexandros Gezerlis, and Sanjay Reddy}

Theoretical Division, Los Alamos National Laboratory, Los Alamos, New Mexico 87545, USA

E-mail: carlson@lanl.gov, gezerlis@lanl.gov, reddy@lanl.gov

We report on recent work on the equation of state and pairing gap of neutron matter and cold atomic systems. Results of quantum Monte Carlo calculations show that the equations of state are very similar. The neutron matter pairing gap at low densities is found to be very large but, except at the smallest densities, significantly suppressed relative to cold atoms. We also discuss recent attempts to measure and extract the pairing gap in the fully paired superfluid state at unitarity.

8th Conference Quark Confinement and the Hadron Spectrum September 1-6 2008

Mainz, Germany

\footnotetext{
* Speaker.
} 


\section{Introduction}

Systems of ultracold Fermi atoms provide a test bed for atomic and nuclear many-body theory. These systems, like the low-density neutron matter found in the crusts of neutron stars, are very strongly paired, with gaps of the order of the Fermi energy, rather than the very small fraction typical in traditional superfluids or superconductors. Strongly paired fermions are important in many contexts: cold Fermi atom experiments, low-density neutron matter, and QCD at the very high baryon densities potentially found in the center of massive neutron stars. Developing a quantitative understanding of strongly paired Fermi systems is important since they offer a unique regime for quantum many-body physics, relevant in very different physical settings, including the structure and cooling of neutron stars. Constraining neutron matter properties can also be important in understanding the exterior of neutron-rich nuclei by constraining parameters of nuclear density functionals. Cold-atom experiments can provide direct tests of the equation of state and the pairing gap in the strongly paired regime, and hence provide a crucial benchmark of many-body theories in these systems.

\section{Equation of state and pairing gap}

For both cold atoms and neutron matter, we consider a system of two fermionic species and a simple Hamiltonian (for a refinement, see the last section) of the form

$$
H=-\frac{\hbar^{2}}{2 m} \sum_{i} \nabla_{i}^{2}+\sum_{i<j} v\left(r_{i j}\right),
$$

where $i$ and $j$ represent spin up and down particles, respectively. In ultracold Fermi gases, the interaction $v(r)$ can be tuned through Feshbach resonances to be very attractive, and to produce a specific scattering length. In this work we are interested in values of the Fermi momentum $k_{F}$ times the scattering length $a$ from -1 to -10 (the BCS side of what is known as the "BCS-BEC crossover") and also $-k_{F} a=\infty$, a universal regime known as unitarity. For these systems, the effective range $r_{e}$ between the atoms is nearly zero.

On the other hand, in low-density neutron matter the scattering length is very large ("unnaturally large"), $\approx-18.5 \mathrm{fm}$, much larger than the typical separation between neutron pairs. The effective range is much smaller than the scattering length, $r_{e} \approx 2.7 \mathrm{fm}$, so $\left|r_{e} / a\right| \approx 0.15$, but only at very low densities is the effective range much smaller than the interparticle spacing. We are using the ${ }^{1} \mathrm{~S}_{0}$ channel of the Argonne v18 potential.[1]

We have performed[2] fixed-node quantum Monte Carlo (QMC) calculations for both cold atoms and neutron matter. In each case, the trial wave function is taken to be of the Jastrow-BCS form with fixed particle number and periodic boundary conditions:

$$
\Psi_{T}=\left[\prod_{i<j} f\left(r_{i j}\right)\right] \mathscr{A}\left[\prod \phi\left(r_{i j}\right)\right] .
$$

The BCS pairing function $\phi(r)$ is parametrized with a short- and long-range part as in Ref. [3]. The fixed-node approximation ensures that the result that follows from such a calculation will be 


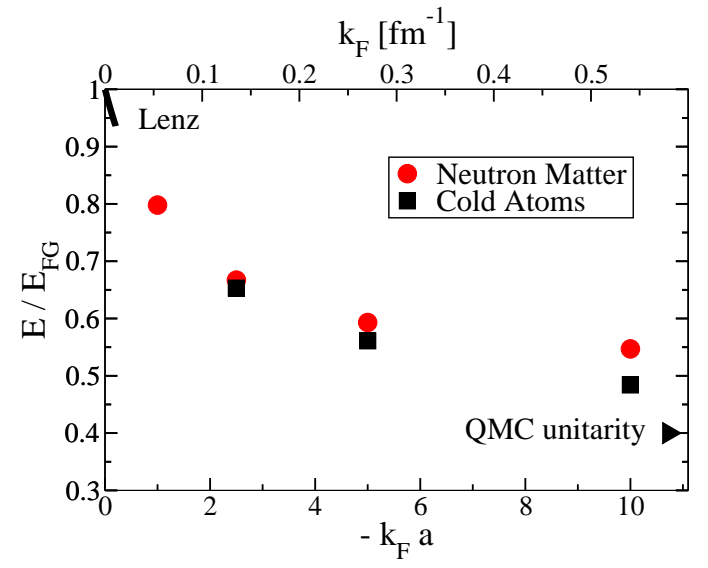

Figure 1: Equations of state at $T=0$ for cold atoms and neutron matter. Also shown are the low-density analytical expansion of the groundstate energy of a normal fluid, and the cold atom result at unitarity ( $k_{F} a=\infty$, arrow). QMC calculations are shown as circles and squares for neutron matter and cold atoms, respectively.

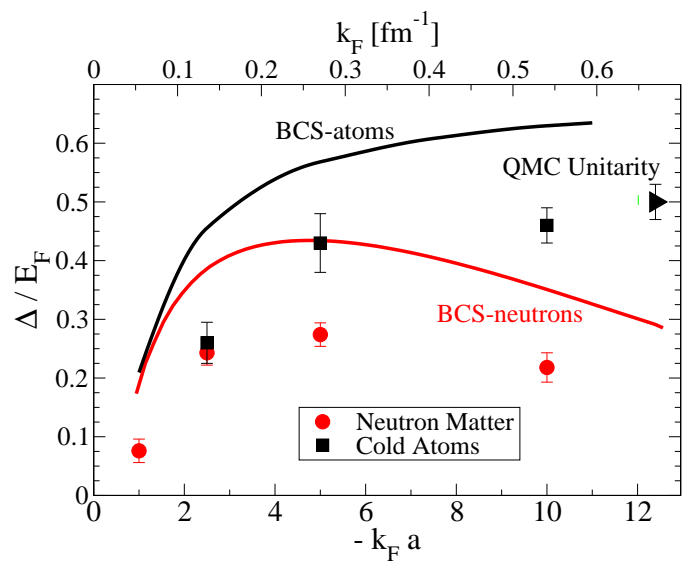

Figure 2: Superfluid pairing gap versus $k_{F} a$ for cold atoms $\left(r_{e} \approx 0\right)$ and neutron matter $\left(\left|r_{e} / a\right| \approx\right.$ $0.15)$. Shown are the mean-field BCS results for both cases (solid lines) and the QMC calculations (points), as well as the cold atom result at unitarity $\left(k_{F} a=\infty\right.$, arrow).

an upper bound to the true ground state energy. We have used this fact to variationally optimize the parameters in $\Psi_{T}$ so as to obtain the lowest possible fixed-node ground-state energy.

In Fig. 11 we compare the $T=0$ equations of state for cold atoms and neutron matter. The horizontal axis is $k_{F} a$, with the equivalent Fermi momentum $k_{F}$ for neutron matter shown along the top. The vertical axis is the ratio of the ground-state energy to the free Fermi gas energy $\left(E_{F G}=3 / 5 E_{F}\right)$ at the same density; only at very low densities (where we are near the limit of a non-interacting gas) is this ratio one. The curve at lower densities shows the analytical result for normal matter: $E / E_{F G}=1+\frac{10}{9 \pi} a k_{F}+\frac{4}{21 \pi^{2}}(11-2 \ln 2)\left(a k_{F}\right)^{2}$, which is only accurate for very low densities.

The equations of state for cold atoms and neutron matter are practically identical at low densities, and are very similar even at densities where the effective range is comparable to the interparticle spacing. This shows why cold atomic systems are a "test bed" for nuclear physics: a measurement of the ground-state energy of, say, a ${ }^{6} \mathrm{Li}$ gas provides results that are directly related to the equation of state of neutron matter. The largest density at which we performed a calculation for neutron matter (since beyond that point non S-wave contributions become considerable; again, see the last section) is at $k_{F} a=-10$. The energy at that point is not too far from QMC calculations [3, 4, 5] and measurements (see Ref. [6]) of the ratio $\xi$ of the unitary gas energy to $E_{F G}$; previous calculations give $\xi=0.42(1)$. Extrapolations of recent QMC calculations to $r_{e}=0$ and also AFMC calculations suggest that $\xi=0.40$ (1) (arrow in Fig. 1).

The pairing gap is the other fundamental zero-temperature property of superfluid systems. Calculations of the pairing gap in many-body simulations are significantly more difficult than the ground-state energy, essentially because the pairing gap is much more susceptible to finite-size truncation errors than the ground- state energy. To examine these more concretely, we have com- 
pared calculations of the BCS pairing gap in finite volumes with periodic boundary conditions to the infinite volume limit. Details of this process of reaching the thermodynamic limit are given in Ref. [2]. The main result is that at least $\mathscr{O}(60)$ particles are needed for such a simulation to be reliable.

Calculations of the s-wave pairing gap in neutron matter have varied enormously over the past 20 years [7, 8]. The difficulties in accurately calculating corrections to the BCS pairing gaps in the strongly paired regime are significant, and hence calculations of the pairing gap can differ by large factors (from 4 to 10) in the low-density regime. Cold atom experiments can provide a critical test of theories of the pairing gap in this regime.

We calculate the pairing gap from the odd-even energy staggering:

$$
\Delta=E(N+1)-\frac{1}{2}(E(N)+E(N+2))
$$

where $N$ is an even number of particles. In Fig. 目 we plot the pairing gap as a function of $k_{F} a$ for both cold atoms and neutron matter.

For very weak coupling, $-k_{F} a<<1$, the pairing gap is expected to be reduced from the BCS value by the polarization corrections calculated by Gorkov [9] $\Delta / \Delta_{B C S}=(1 / 4 e)^{1 / 3}$. Because of finite-size effects, it is difficult to calculate pairing gaps using QMC in the weak coupling regime. The QMC calculations at the lowest density, $k_{F} a=-1$, are roughly consistent with this reduction from the BCS value. At slightly larger yet still small densities, where $-k_{F} a=\mathscr{O}(1)$ but $k_{F} r_{e}<<1$ for neutron matter, one would expect the pairing gap to be similar for cold atoms and neutron matter. The results at $k_{F} a=-2.5$, where $k_{F} r_{e} \approx 0.35$, support this expectation. Beyond that density the effective range becomes important and the QMC results are significantly reduced in relation to the cold atoms where $r_{e} \approx 0$. Such a microscopic calculation of the neutron matter ${ }^{1} \mathrm{~S}_{0}$ pairing gap is potentially important to neutron star cooling and to Skyrme-Hartree-Fock-Bogoliubov mass formulas.[10].

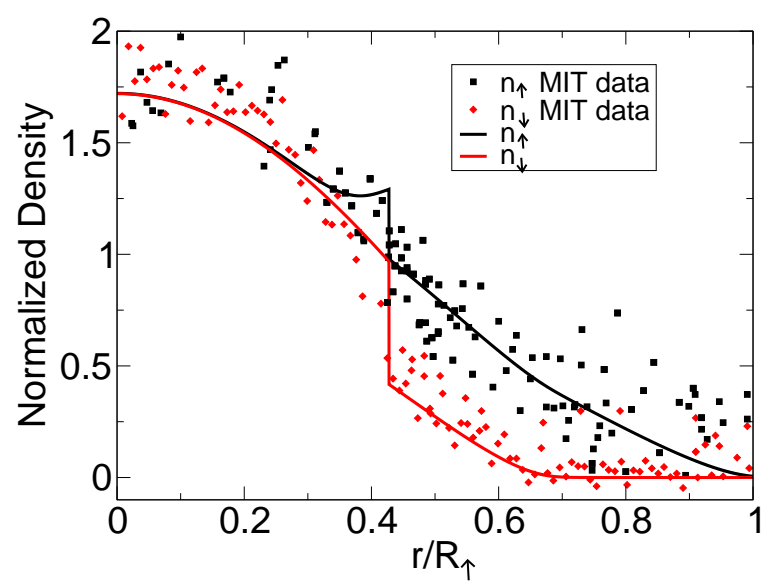

Figure 3: Experimental points and theoretical curves for the spin-up and spin-down densities as a function of distance from the center of the trap. 
Moving on to the experimental situation, measurements of the local polarization in the trap provide direct information on the pairing gap. In such experiments the spin-averaged local chemical potential is fixed by the trap potential and the total number of particles. The difference between the up and down chemical potentials is independent of the position in the trap. At zero temperature in a slowly-varying potential the system will separate into three regions: a superfluid region in the center which has been found to have zero net polarization, a normal polarized region in the middle with different numbers of spin up and spin down particles, and a fully polarized region in the exterior consisting of only one species. The fact that the interior region is unpolarized can be used to place a lower bound on the pairing gap (see Fig. 3). At finite temperature the interior superfluid will be polarized by thermal effects, and measurements of the polarization as a function of position in the trap provide a sensitive estimate of the pairing gap. We have analyzed recent measurements by the MIT group[11] and obtained an estimate for the pairing gap that is $0.45(5) E_{F}$ in the unitary regime. [12] This can be compared with the many-body calculation shown in Fig. 2 which is $0.50(3) E_{F}$ and also with the recent experimental result [13] which is $0.44(3) E_{F}$.

\section{Ongoing work}

Apart from the equation of state, it is possible to calculate other quantities using QMC, like the momentum distribution, the pair distribution function, and the quasiparticle dispersion. Furthermore, it is possible to use a potential that is more refined than the one used in Ref. [2], by incorporating more terms from the AV18 potential, i.e.

$$
v_{4}(r)=v_{c}(r)+v_{\sigma}(r) \sigma_{1} \cdot \sigma_{2}
$$

The quasi-particle spectrum that results from such an approach is shown in Fig. \#; it is calculated by putting the unpaired particle in different momentum states and using Eq. (2.3). A knowledge of this quantity is relevant to calculations of the spin susceptibility of neutron matter, which are of interest in neutron star physics. Further details and results for these quantities with this new potential are to be provided in a forthcoming publication.[14].

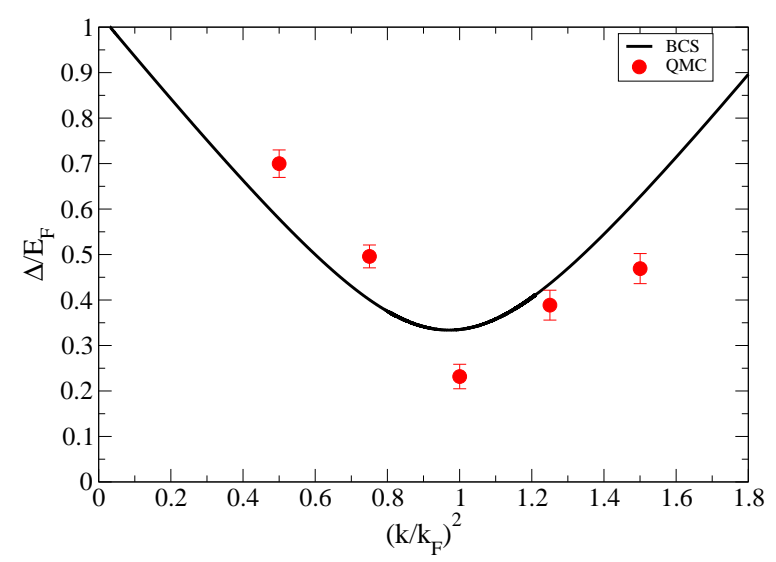

Figure 4: Neutron matter quasi-particle dispersion in BCS (line) and GFMC (points) at $k_{F} a=-10$. 
Another direction such calculations have taken is related to the physics of cold atoms: it is possible [15, 16] to trap Fermi-Fermi mixtures (e.g. of ${ }^{6} \mathrm{Li}$ and ${ }^{40} \mathrm{~K}$ ) in the laboratory and also to tune the relative populations of the two species. There is no a priori reason to expect such an unequal-mass system to behave in exactly the same way as the corresponding equal-mass one (though in mean-field theory that is the case), i.e. the possibility of exotic states has to be considered. In a forthcoming publication[17] we give results for the quasiparticle spectrum as well as the energy as a function of polarization for such a system.

\section{Acknowledgments}

The work of the authors is supported by the Nuclear Physics Office of the U.S. Department of Energy and by the LDRD program at Los Alamos National Laboratory. The work on neutron matter is supported by the UNEDF SCIDAC program of the U.S. Department of Energy. Computing resources were provided at LANL and NERSC through the LANL Open Supercomputing Program and SCIDAC.

\section{References}

[1] R. B. Wiringa, V. G. J. Stoks, and R. Schiavilla, Phys. Rev. C 51, 38 (1995).

[2] A. Gezerlis and J. Carlson, Phys. Rev. C 77, 032801(R) (2008).

[3] J. Carlson, S. Y. Chang, V. R. Pandharipande, and K. E. Schmidt, Phys. Rev. Lett. 91, 050401 (2003).

[4] J. Carlson and S. Reddy, Phys. Rev. Lett. 95, 060401 (2005).

[5] G. E. Astrakharchik, J. Boronat, J. Casulleras, and S. Giorgini, Phys. Rev. Lett. 93, 200404 (2004).

[6] S. Giorgini, L. P. Pitaevskii, and S. Stringari, Rev. Mod. Phys. 80, 1215 (2008).

[7] U. Lombardo and H. -J. Schulze, Lecture Notes in Physics (Springer-Verlag, Berlin, 2001) Vol. 578, p. 30 .

[8] D. J. Dean and M. Hjorth-Jensen, Rev. Mod. Phys. 75, 607 (2003).

[9] L. P. Gorkov and T. K. Melik-Barkhudarov, JETP, 40, 1452 (1961) [Soviet Phys. JETP 13, 1018 (1961)].

[10] N. Chamel, S. Goriely and J.M. Pearson, Nucl. Phys. A812, 72 (2008).

[11] Y. Shin, C. H. Schunck, A. Schirotzek, and W. Ketterle, Nature 451, 689 (2008).

[12] J. Carlson and S. Reddy, Phys. Rev. Lett. 100, 150403 (2008).

[13] A. Schirotzek, Y. Shin, C. H. Schunck, and W. Ketterle, Phys. Rev. Lett. 101, 140403 (2008).

[14] A. Gezerlis and J. Carlson, to be submitted.

[15] M. Taglieber, A.-C. Voigt, T. Aoki, T. W. Haensch, and K. Dieckmann, Phys. Rev. Lett. 100, 010401 (2008).

[16] E. Wille, et al., Phys. Rev. Lett. 100, 053201 (2008).

[17] A. Gezerlis, S. Gandolfi, and J. Carlson, to be submitted. 